\title{
菌物在森林生态系统中的功能和作用研究进展
}

\author{
严东辉 ${ }^{12}$ 姚一建 ${ }^{\text {* }}$ \\ (1 中国科学院微生物研究所, 100080 北京) \\ (2 中国林业科学研究院森林生态环境与保护研究所, 100091 北京)
}

\begin{abstract}
摘 要 综述了菌物在森林生态系统中的功能和作用方面的研究进展, 表明菌物是森林生态系统中不可忽视的重 要组成部分，对森林生态系统的建立、演替、稳定、物质循环和能量流动等都具有重要的影响。菌物与植物的共生 是植物起源和森林形成的基础, 而且是森林健康发展的关键; 菌物在森林中的分解作用和对土壤母岩的风化作用 是生物地球化学循环的重要环节, 且菌体本身是森林中的主要碳、氮汇成员; 菌物多样性是森林物种多样性和生态 稳定的决定因素之一, 其多样性的丧失可以直接影响森林的生态系统乃至加剧地球的大气温室效应。菌物在我国 森林保护和可持续发展以及生态环境建设上具有重要的位置。
\end{abstract}

关键词 菌物多样性 森林生态 森林保护 菌根

\section{RECENT ADVANCE OF RESEARCH ON FUNGI IN FOREST ECOSYSTEM}

\author{
YAN Dong-Hui ${ }^{1}{ }^{2}$ and YAO Yi-Jian ${ }^{1 *}$ \\ (1 Institute of Microbiology, the Chinese Academy of Sciences, Beijing 100080 , China) \\ (2 Institute of Forest Ecology , Environment and Protection, the Chinese Academy of Forestry, Beijing 100091 , China)
}

\begin{abstract}
The recent advance of research on fungi in forest ecosystem is reviewed in this paper. It is shown that fungi are important components in forest ecosystem with their roles in establishing plant community , promoting succession of species population , retaining forest ecosystem stability and participating in the flow of energy and nutrition. The early colonization of land by plants was associated with fungi. Evidences have shown that the symbiosis between plants and fungi was established when the terrestrial life started. By co-evolution with plants , fungi are involved in the formation of the complex forest ecosystem. Mycorrhizae have apparently played an important role in the origin of terrestrial plants and they are the key to the development of forest. Mycorrhizal fungi are crucial not only to phosphorus uptake by plants, but also to other mineral nutrition. Decomposition of organic material and weathering of mine by fungi in forest contribute greatly to the biogeochemical and nutrient cycles on earth. Large biomass of fungi in forest forms important $\mathrm{C}$ and $\mathrm{N}$ sinks. Abundance and diversity of fungi in forest has significant influences on biodiversity and stability of forest community. Loss of fungal diversity may instable the forest ecosystem and, further, aggravate the greenhouse effects of the world. Enforcing research on fungal roles in forest ecology will facilitate conservation and sustainable development of forest and the forest ecological projects in China.
\end{abstract}

Key words Fungal biodiversity , Forest ecology , Forest conservation , Mycorrhiza

森林生态系统的保护和建设是环境保护的重要 内容,全球灾害性气候频发,生物多样性丧失, 温室 效应加重等环境问题都与森林生态的破坏密切相 关。为此,世界各国十分重视森林对环境保护的作 用。在我国 环境保护和森林生态建设已成为基本 国策，近年来投入了巨额资金开展林业生态工程建 设。从 2001 年开始全面实施的六大国家级林业工 程, 力图今后 10 年内在天然林保护、退耕还林 (还 草)、长江中下游等防护林体系建设、防沙治沙、野生
动植物保护和速生丰产林基地建设等领域取得突破 性进展，实现中国林业的跨越式发展，使中国的生态 环境得到明显改善(News Centre, the Chinese Academy of Forestry, 2001)。为实现这一目标, 很有必要对森 林生态系统中各重要组成要素进行科学研究, 正确 认识它们之间的相互关系。随着生命科学和生态学 的迅速发展, 菌物作为森林生态系统中的植物共生 生物、有机质分解力量和生物多样性的重要组成成 分越来越广泛地得到认识。菌物在森林生态系统的 
建立、演化和稳定发展等过程中具有不可忽视的功 能, 有时甚至起决定性的作用。本文就近年来有关 菌物在森林生态中的功能和作用的研究进展作一概 述, 同时指出深刻认识和研究菌物在当前我国森林 保护和可持续发展中的重要意义。

菌物 (Fungi) 在传统上被定义为具有真核而无 叶绿素, 行吸收营养的有机体, 普遍以有性和无性两 种方式进行繁殖，产生敄子，通常为丝状且有分枝的 体细胞结构, 典型地为细胞壁所包裹。在历史上, 这 类生物曾被划分为植物界的一部分。现在对菌物的 认识已超越了上述以形态特点和营养方式为主的定 义, 而在细胞学、生物化学、分子水平上都有了新的 突破和解释 (Alexopoulo et al. , 1996)。菌物是独特 的，在生物的界级分类上与动物界、植物界并列已成 为共识。而且 根据 DNA 数据的系统发育研究结果 还认为菌物不与植物, 而与动物构成姊妹系 (Hecht, 1993 ;Alexopoulo et al. 1996)。在系统发育上, 菌物 的这一传统定义所界定的实际上是一个多系的 (Polyphyletic)生物类群, 包括了一些现在已经明确 的、不是真正菌物的有机体。由于这些生物到目前 还是由菌物学家们来研究的, 所以我们在这里采用 的还是广义的菌物概念 (Hawksworth, 1991; ;lexopoulo et al. , 1996)。

\section{1 菌物对植物起源和森林形成的重要作用}

植物起源于地球生命初期的光合单细胞生物, 经水生到陆生、单细胞到多细胞、简单到复杂、低等 到高等的演化和适应(郝守刚等，2000）, 形成了现今 27 万余种的植物界 (Gleich et al. ,2001)。而远古光 合生物能够成功地登陆可能就离不开菌物的帮助 (Pirozynski \& Malloch ,1975;Heckman et al.,2001)。 陆地植物和菌物共生的最早化石记录是在 4 亿 6 千 万年到 4 亿 8 千万年之间 ( Redecker et al. ,2000； Heckman et al. 2001), 而种子植物的出现则是在 4 亿 3 千万年前的古生代志留纪 (Heckman et al. , 2001)。近年来在分子水平上对菌物的起源、菌物与 植物的进化关系进行的研究上获得了更多的证据, 表明菌物和植物的共生关系早在陆地生命初期就开 始建立了 (Gargas et al .,1995 ;Jongmans et al. ,1997 ; Berbee \& Taylor , 2000 ; Blackwell , 2000 ; Heckman et al. 2001;Lutzoni et al. 2001;Pennisi 2001)。基于核 糖体基因分子钟的推测, 菌物的大量分化大约出现 在 6 亿年前的前寒武纪晚期 (Berbee \& Taylor 2000)； 而以一百多种蛋白序列进行的分析则认为菌物和光
合生物远在 10 亿年前就已开始建立共生关系 (Heckman et al.,2001;Pennisi,2001)。菌物不仅分 解有机物, 并且还参与岩石的风化分解, 从中离析和 汲取矿物元素供给植物 (Jongmans et al. 1997)。菌 物的这一营养方式满足了初始登陆植物的基本营养 需求, 并使植物能适应陆地的生境而在各种生态条 件下成功地定植下来 (Blackwell ,2000)。现存的菌物 和光合生物共生关系的主要方式是地衣和菌根, 地 衣是菌物和低等藻类的共生体, 是岩石风化形成土 壤的先锋生物和植物分布的先导, 而菌根共生体则 是绝大多数陆地植物地下根系的不可缺少的组成部 分。在地衣共生体中, 菌物构成了可供辨认的主要 部分。虽然有人认为地衣中的菌物成员 (主要是子 囊菌)有着不同的起源 (Gargas et al.,1995), 但现在 有证据表明它们可能都起源于一个共同的祖先, 而 大量的菌物是通过非地衣化的过程而演化出来的 (Lutzoni et al. 2001)。这说明地衣共生关系在帮助 植物登陆, 为其定植创造条件的同时, 也为菌物自身 的登陆和分化奠定了基础。菌物与光合生物的协同 关系造就了今天地球陆地森林生态的多样性。许多 陆地森林生态系统的重要建群树种及其所在科, 如 桦科 (Betulaceae)、松科 (Pinaceae) , 水青冈科 ( Fagaceae) 和龙脑香科 (Dipterocarpaceae) 等, 都是专性菌 根植物 (Smith \& Read ,1997)。这些树木离开了菌根 菌几乎不能生长, 更谈不上建立种群并与其它物种 组成群落。虽然植物种的起源尚有准确的解释, 目 前也缺乏菌物如何帮助植物建种建群并演化成森林 的直接证据，但根据现有菌物与植物共生关系的普 遍性和对森林植物的重要性, 还是可以较为肯定地 推论菌物帮助植物成功地登了陆 (Pirozynski \& Malloch, 1975;Blackwell ,2000) ,并与早期植物协同进化， 从而形成了陆地上最重要和最复杂的主体生态系统 森林。

\section{2 菌物对森林生态中物流和能流的重要作 用}

森林生态中的物质循环和能量流动是生物地球 化学循环中碳、氮循环的中心环节, 也是生物圈和地 层矿物营养交流的主体。植物生长受制于土壤无机 营养的可利用性, 营养的获取和供给是控制植物生 产率和多样性最重要的两个因素 (Pugnaire,2001)。 菌物通过改变森林土壤营养来源的可利用性和影响 森林种群营养的相互关系, 调节和影响森林生态系 
统的进程 (Chapin III et al. 1997)。

\section{1 菌物对森林生态中磷、氮、碳营养的作用}

植物生长代谢需要的磷大部分是由菌根菌提供 的 磷在菌根菌和植物根系营养代谢的交流中处于 中心位置 (Pfeffer et al . 2001)。菌根菌代谢的多磷 化合物 (Polyphosphate) 控制菌根中无机正磷酸 (Pi) 的流向 进而调节可利用的无机正磷酸传输到树木 生长旺盛的需要点上(Pfeffer et al.,2001)。菌根中 至少存在两个磷库:一个是由菌物传输到植物去的、 相对较小的磷酸盐库; 另一个是相对无活性的、较大 的、可以累积的多磷化合物颗粒库 (Harley \& Smith， 1983)。后者是前者的储备, 菌根菌从土壤中吸收正 磷酸, 经形成较短链的可移动的多磷物 $(n \leqslant 100)$, 再形成储藏态多磷物, 当需要时, 多磷化合物水解成 可利用态磷供给植物 (Gerlitz \& Werk, 1994)。因此， 在缺磷的立地条件下, 通过菌根菌的磷吸收, 可以促 进植物的生长 (MacFall et al , 1992)。

菌根菌的磷代谢同时促进了植物的氮代谢作用 (Pfeffer et al . 2001) 经常发现具有菌根的树种对周 围植物的生长发育具有类似于施用氮肥的效果, 这 可能与菌根菌同化土壤中的铵盐有关 (Finlay et al. , 1998)。菌根由精氨酸途径代谢来自于土壤的铵盐, 菌根中精氨酸和多磷化合物代谢是相关联的 (Pfeffer et al. 2001)。在菌根和根瘤联合共生的植物 (如杞 木( Alnus incana) 和木麻黄 (Casurina equisetifolia)) 中 植物通过菌根改善了磷营养的供给 增强了固氮 反应中动力酶的作用, 从而促进根瘤的固氮作用和 提高了树木的抗性(Reddel et al. ,1997)。对菌根菌 通过联合代谢供给和调节植物所需的氮和磷的机 制现在有了更具体和明确的阐明 (Martin,1985; MacFall et al. 1992;Pfeffer et al. 2001)。此外, 菌根 菌也是地球上重要的氮汇之一, 研究观察表明菌物 比植物含有更高的氮丰度 (Taylor et al , 1997)。从 森林中不断消亡的有机体上游离出来的氮等元素， 通过菌根的迅速吸收固定，可重新被植物利用 (Naeshom et al. 1998)。

除了对磷、氮的作用之外，菌根共生菌物对森林 碳利用的影响则具有更重要的生态学意义, 其主要 表现在森林能量合成和能流配置上(见 2.3 节)。还 有以其它方式进行生活的菌物也对森林中碳素的营 养具有重要的作用。营腐生方式的菌物是地球上最 大碳源的纤维素、木质素和几丁质类物质的主要分 解者 (Alexopoulo et al ，2002)。菌物释放碳素和碳 汇调节的作用对地球碳化学循环不可或缺。并且，
菌物对森林中大量的病、弱、死树和枯枝落叶的分解 作用与森林的生长直接相关, 分解释放的营养元素 以及这些营养元素返回森林土壤的速率决定了森林 的生长率(冯宗炜等 ,1999; Alexopoulo et al . ,2002)。

\section{2 菌物对森林中其它矿物营养的作用}

菌物在贫㾑的土壤状态下为植物矿物营养元素 提供了有效的吸收和传输系统 (Marschner \& Dell , 1994)。根据 Arocena 和 Glowa (2000) 的研究, 外生菌 根菌 (Piloderma spp.) 能使亚高山冷杉 (Abies lasiocarpa )根围土壤溶液中的营养矿物元素浓度远远高 于根围以外的土壤。菌根菌的外部菌索通过其表面 对土壤物质的吸收，可以为宿主植物提供 $10 \%$ 的 钾、 $25 \%$ 的锌、 $60 \%$ 的铜，以及其它满足植物基本代 谢的镁、硼、铁等微量营养元素 (Marschner \& Dell, 1994）。研究还表明共生菌根菌能以腐生方式主动 将矿化的元素转化为可利用的状态, 以补充和保障 植物的基本营养需求 (Jongmans et al. 1997; Hobbie et al. 2001)。Jongmans 等 (1997)观察到菌根菌在菌 丝顶端分泌柠檬酸、琥珀酸、草酸、甲酸和苹果酸等 有机酸, 以每年 $0.3 \sim 30 \mu \mathrm{m}$ 的速度在矿石上溶解出 许多小洞, 洞液中含有比周围浓度高出很多的多种 矿物营养元素。他们观察到土壤与基岩之间的区域 是菌根菌菌丝分布最密集的地方, 而且点柄乳牛肝 菌 (Suillus granulatus) 和另一种菌根菌 (Piloderma croceum) 的菌丝直接将矿石与植物根系相连在一起， 通过菌丝传输 将溶解出的元素直接供给植物利用。 菌物主动分解和为植物提供矿物质的过程, 有助于 说明近年来发现的森林土壤虽然过度酸化, 但未影 响到森林生产率的现象 (Jongmans et al .,1997)。

在酸性土壤条件下, 容易发生矿物营养元素束 缚和铝元素干扰树木矿营养生理和功能等有害作 用, 菌根菌的存在能有效地保障树木从母岩中汲取 必需的矿物质和抵御这些有害作用 (Jongmans et $a l$ ，1997)。与不具有菌根共生体的植物相比, 菌根 菌可以为植物带来高出几倍的矿物营养浓度 (Clark et al. 1999)。菌根树种对铝害具有较高的抵抗能 力 (Gerliz,1996)，原因是菌根通过磷代谢途径产生 的多磷化合物可謷合铝元素而使其毒害减低或消失 (Martin et al. ,1994)。菌根的这些作用，促进和保障 了植物在不良立地条件下正常生长 (Martin et al. , ; Gerliz, 1996; Clark et al . 1999 ; 1996 ; 2000)。菌物能主动分解并提供矿物元素, 而且抑制 有害元素的作用也许是植物离不开菌物的又一重要 原因。 


\section{3 菌物对森林生态中能流的作用}

在能源分配和平衡上, 共生菌根菌为植物提供 氨基酸和矿物营养 (Martin,1985) ,而植物则为菌物 提供光合产物作为回报 (Martin et al. ,1994)。植物 的光合产物有一半以上从地上输送到地下部分, 用 于根系的生长和根围生物群落的生存和利用 (Setala et al . 1999) ,而菌根共生活动利用的光合产物可达 30\%左右( Söderström, 1992 ;Finlay \& Söderström ,1998; Nehls et al. ,2001)。也许是因为这个原因, 菌根的 共生能促进寄主光合作用和增加同化产物 (Lowe et al. 2000)。

森林植物在地下形成的庞大和复杂的菌根菌生 物量是森林的巨大碳汇 (Hogberg et al , 1999 ;Bidartondo et al . 2000; ;Hogberg et al . 2001; ;Nehls et al . , 2001)。菌根菌、植物根系及其它异养微生物的呼吸 活动构成了地球碳平衡的重要部分，从松林地上部 分阻断光合产物向根部输送，几天之内土壤呼吸即 下降 37\% (Hogberg et al.,2001)。植物同化碳的过 程主要受到决定光合产物分配流向的碳汇强度的影 响，碳汇强度控制着植物光合同化率，碳素总是直接 流向最具活性的汇区(Stitt ,1991)。菌根共生能产生 重度的碳饥渴，促使高达 30\% 的光合产物转移到共 生活动中, 以供给菌根菌使用 (Finlay \& Söderström , 1998)。在菌根菌生长期, 菌根菌将大气循环中的碳 通过植物进入菌物体中, 如菌根菌通过庞大的菌丝 系统将碳素以能量载体的形式传送到子实体上,用 于子实体的形成和发育 (Hogberg et al.,2001)。有 实验表明 (Lamhamedi et al .,1994) ,摘去双色蜡蘑 ( Laccaria bicolor) 的子实体或抑制其生长发育能降 低宿主植物的净光合产物的流速和减低净光合率。 同时, 不同组群以及在不同的外界生长因子条件下 的菌物，可通过自身组织的生产、生活和分解来影响 碳的能流循环, 如菌物种间、种内的不同分离株系具 有巨大反差生产率的性质就说明了这一点 (Cairney , 1999)。此外，已有证据表明(Burke \& Cairney , 1997 ; 1998 ) 菌根菌还能产生碳水化合物氧化酶，以分解环 境中的木质纤维素, 对自然界中碳的循环起着更积 极的作用。

菌根菌的菌丝在地下相互连接而形成的网络 ( David ,1997 ;Simard et al.,1997 ; Helgason et al. , 1998 ;Dahlberg，2001），能使森林中的能源物质能够 向最需配置碳素的地方输送 (Nehls et al.,2001)。 光合同化作为植物的基本代谢, 是植物监测和调节 糖水平变化来适应外部环境的主要生化过程 (Nehls et al，2001）植物通过菌根菌在树种的种内和种间 进行相互传递来调节整个森林能流的源与汇 (Simard et al. ,1997 ;Smith \& Read,1997 ; Rogers et al. 2001; ; w et al. ,2001) ,从而维护森林生态能量 的平衡和保持食物链中能量的有效流动。由此可 见, 菌物在林木种间互作、森林多样性的维持、森林 生态系统的稳定性、生产率和可持续利用，以及生物 地球化学循环、甚至改善地球温室效应上的作用是 十分重大的。

\section{3 菌物维系着森林生态系统的稳定和发展}

目前估计地球上有 150 万种菌物，而被认识的 仅有 7 万种左右 (Hawksworth, 1991;Kohn, 1999)。然 而，在这些已知的菌物中，已证明有的种类能够形成 陆地上最大的生物个体。美国太平洋东北林业站的 科学家在奥勒冈 (Oregon) 东部 Malheur 国家森林里 发现的奥氏密环菌 (Armillaria ostoyae) 的巨型菌体横 跨 3.5 英里, 其所占据的地面面积约为 1665 个足球 场，并在地下达到平均 3 英尺的深度，估计的年龄约 为 2400 年 (Pacific Northeast Research Statin/USDA Forest Sevice 2000)。除了其个体可与鲸鱼、巨红杉 和芒松等大型的动物、植物相比外, 这种巨菌对森林 的作用也是引人注目的 (Alexopoulo et al. ,2002)。 菌物在森林生态系统中以腐生、共生、寄生、内生等 生活方式与植物和环境发生作用，这些作用已被认 识到是森林生态中的基本功能, 如上述参与森林地 球化学循环中生物功能的能流和物流、主动风化和 分解释放矿化的营养物质等。此外，对菌物在参与 决定、创建和稳定森林生态系统中物种多样性的作 用研究有了进一步的了解。

3.1 菌物对森林植物多样性及森林生态稳定的作 用

热带雨林中的树种多样性是由于一些生物的作 用，主要是由依赖某单一树种 (树种专化性) 而生活 的植食性生物所造成的, 尤其是植食性昆虫与其取 食对象树种间的密切关系起着非常关键的作用 (van der Putten,2000)。早在 20 世纪 70 年代, Janzen (1970) 就认为因为昆虫同时取食成熟的大树和幼 苗 幼苗由于抵抗能力弱, 易被昆虫取食而消亡, 所 以幼苗难以在同种母树附近生存, 而其它种类的树 木则可在临近地得以生存和发展, 这样就形成了热 带雨林树种的多样性。近来在亚热带林的土壤中， Packer 和 Clay (2000) 发现植物病原菌物具有类似于 植食性昆虫在热带雨林中驱动多样性的作用。定殖 
于黑桃 (Prunus serotina) 根上的腐霉菌 (Pythium sp.) 能感染致死母树周围的同种小苗, 使母树附近 $15 \mathrm{~m}$ 以内的区域生长出不同的树种 (Packer \& Clay， 2000)。因此，森林土壤中宿存的树木天敌也是创造 森林种群和保持物种多样性的重要和关键因素 ( Janzen ,1970 ; Packer \& Clay ,2000 ; van der Putten , 2000)。

菌物与植物的共生关系是森林生态系统的稳定 和发展的基础。在植物定植伊始, 菌根菌就与植物 形成了动态的菌根共生体系。菌根菌保护树木不受 病原菌的侵袭, 提供树木生长需要的 $\mathrm{N} 、 \mathrm{P} 、 \mathrm{~K}^{+}$、 $\mathrm{Ca}^{2+}$ 、酶、激素等生长因子，维护和促进树木和种群 的健康发展。菌根的菌丝将森林中的树木个体联系 形成一个整体, 从而使森林成为复杂的营养和功能 互动的生态网, 其中每一成员都能共享森林的物质 资源 ( David ,1997; Simard et al.,1997;Helgason et al. , 1998; Dahlberg, 2001) 维护着森林群落的生态平 衡。如森林外围阳光充裕地带的北美白桦 (Betula papyrifera) 有 $6 \%$ 的光合产物通过菌根菌的传输, 为 处在森林里阳光相对较少的地方、光合作用弱的花 旗松 ( Pseudotsuga menziesii) 所利用 (Simard et al. , 1997)。可以认为, 这一作用使森林深处的其它低矮 种类和幼树在缺少光合效率的条件下得以生存和建 立种群, 这也说明森林群落中的种群之间的关系并 不只是生存竞争的关系, 还有互相依存的关系 (David ,1997)。Dahlberg(2001) 还认为, 如果网络传 递主要是从先锋植物向后续物种输送的话, 那么菌 根菌可能就是决定植物群落发展的一个重要因素。

菌物多样性可以看作是生态稳定的指标之一， 菌根菌种类在脆弱的农田生态系统中是非常有限 的 种类比较少 (Helgason et al , 1998) , 而在良好的 森林生态系统中, 菌物的多样性则往往比较丰富。 有研究表明 ( van der Heijden et al .,1998) ,内生菌根 菌的多样性对生态系统功能的优化起到关键性的作 用, 这对 Bergelson 和 Crawley (1988) 提出的菌根菌是 否能影响森林种群多样性的疑问作出了肯定的回 答。长期的研究还表明 (Brown et al. ,2001) ,外生菌 根菌能使森林关键树种松树 (Pinus edulis) 对环境中 危险性生物因子 (蛀干蛾 (Dioryctr albovittella)) 或胁 迫性非生物因素(干旱等)的抗性以及损害的补偿能 力大大提高, 从而维护生态系统的动态平衡和结构 稳定。有一些专营寄生生活的无叶绿素的森林寄生 植物则是依靠菌根菌从其它绿色植物上获取碳源 的, 而并不通过直接的寄生来生存 (Cullings et al.,
1996)。森林中另一类菌物一内生菌的生态作用 也逐步被认识 ( Saikkonen et al. ,1998; Clay \& Holah， 1999)。内生菌物几乎存在于所有的植物中, 使寄主 植物增强生存竞争能力, 并帮助植物抵抗外界不良 因素的干扰(Saikkonen et al.,1998)。最近对专化性 内生菌能改变植物群落结构的作用也被揭示 (Clay \& Holah ,1999) ,而且 Saikkonen 等 (1998) 还认为内生 菌物对森林的种群动态、植物群落多样性和生态功 能等可能都有相当的作用。

植物病原菌物的入侵, 能对森林生物多样性造 成巨大的破坏，如荷兰榆树病、栎枯萎和近期的铠木 疫病(Pain ,1999)。这也从负面的影响说明菌物对森 林生态作用的不可忽视性。

3.2 菌物对森林生态系统中其它物种生物多样性 的维护作用

在森林中, 菌物的物种多样性及其生态是森林 生物多样性和生态的重要子系统, 它们在食物链和 维护生物多样性上起着重要的作用。许多大型菌物 的子实体常常是许多动物的食物，如地下块菌是一 些啮齿动物的主食。欧洲朱松鼠( Red squirrels) 能区 分真假块菌, 并只取食子囊菌的大团囊菌属的成员 (Elaphomyces muricatus) 和更珍贵的种类 (Hydnotrya tulasnei) , 而对非子囊菌的含糊黑腹菌 (Melanogaster ambiguus)、须腹菌属的成员 (Rhizopogon roseolus)、内 囊霉属的成员 (Endogne flammicorona) , 以及一些牛 肝菌等则不感兴趣 (Turnbull ,1995)。一些大型动物 的补充营养也需要食用菌物的子实体。

外生菌根菌能将更多的能量物质导入森林土 壤, 促使地下食物网的交流, 而使土壤生物量增加， 这对土壤肥力的影响是积极的 (Setala et al. 1999)。 菌物生物多样性的减少或丧失，对森林生物多样性 和食物链的影响是不可忽视的。红拟层孔菌 (Fomitopsis rosea) 的数量由于砍伐云杉而减少, 影响了以 此为食的谷蛾 (Agnathosia mendicella) 的种群数量, 进 而影响到寄生于谷蛾上的寄生蝇 (Elfia cingulata) 的 数量(Komonen et al. 2000)。木腐菌在活树上导致 的空洞可以为许多动物提供栖身场所。象牙喙豚木 乌(Ivory billed woodpecker)的绝迹在很大程度上是由 于人类活动干扰了木腐菌的生境, 减少了其赖以生 存的栖身场所而造成的 (Blackwell et al.,2001)。森 林中 1000 多种的鞘翅目的昆虫依靠致病木腐菌或 腐生木腐菌而生存(Komonen et al. 2000) ,而昆虫的 生物多样性是森林种群多样性的关键 (Janzen, 1970)。昆虫共生菌物维持森林昆虫的多样性，二者 
的相互适应和进化是森林生态选择压力的结果, 反 过来也会促使森林生态系统的演替变化 ( Paine et $a l$, 1997) 就如鞘翅目中专蛙性昆虫与其专化伴随 菌物的相互作用就能影响寄主树木的生态位。

\section{4 应当加强我国菌物的生态作用研究}

在森林生态层次对菌物进行的实际研究目前在 我国还很缺乏, 而且还没有引起足够的重视和投入。 我国幅员广阔，森林生态类型十分丰富 (蒋有绪等， 1998a）,许多森林生态中的关键树种都是菌根专性 种。菌根菌的种群生态与森林类型密切关联, 目前 利用 ITS 的 PCR-RFLP 和序列对 50 多种外生菌根菌 的群落结构进行了研究, 并试图从环境基因组学角 度研究菌物群落在相似的森林生态条件下的功能, 以期在分子生态水平上认识菌物种群与植物种群及 其环境的生态互作。我国菌物学的分子系统发育研 究有很强的基础, 可以利用各种分子遗传标记, 开展 主要优势菌物 (菌根菌)种群在重要的森林生态类型 中的分子生态学研究。虽然我国的生态学家还注意 到菌物对森林枯枝落叶的分解作用 (蒋有绪等, 1998b)，以及土壤生物对森林生物生长量的影响(冯 宗炜等，1999），但就菌物与植物的协同进化、菌物与 森林生态类型的关系、菌物多样性对森林生态稳定 的作用、菌物在影响森林生物地球化学循环以及作 为主要碳汇成员调节温室气体等环境生态方面我国 一直很薄弱, 应当加强这些方面的研究。

研究菌物在森林生态中的作用, 对我国的退耕 还林、植树造林等生态恢复和天然林保护的生态工 程建设中也有着重要的现实意义。这就要求我们对 菌根的共生生理和共生的发生生态, 以及森林生态 系统对有害植物病原真菌的正向调控和补偿的机理 进行深入细致的研究。20世纪 70 年代我国国外松 引种初期不太成功的原因就在于忽视了松树是专性 菌根树种(弓明钦等,1997)。目前菌根菌在我国林 业引种、育苗、造林等人工林的建设和生产上已发挥 了重要作用 (弓明钦等, 1997)。菌根菌的磷营养和 根瘤菌的高效固氮作用可以使联合共生植物成为在 贫痊土壤地区生态恢复造林的先锋树种。菌物的菌 根菌除了与植物建立菌根, 帮助树木定植和建林后 外, 还可以成为可持续利用的、具有很高经济价值的 森林非木材产品的重要组成部分。注重先锋树种的 利用和兼顾菌物的非木材产品的效益产出, 对我国 西部森林生态建设和山区开发可起到积极的作用。 很多菌根菌的大型子实体是美味的且价格昂贵的食
用菌, 如松茸 (Tricholoma matsutake)、牛肝菌 (Boletus spp.) 和块菌 (Tuber spp.) 等。许多菌物在食用、药 用以及农林害虫生物防治和工业生产上都有经济开 发利用的价值 (魏江春, 1993)。天然森林中的菌物 多样性是我们现在和将来可以依赖和发现更具利用 价值资源的宝库(魏江春，1993)。菌物能风化分解 母岩直接为植物提供营养, 使人们对森林培育的现 有一些措施( 如施用石灰应付森林土壤酸化) 进行重 新认识和评价 (Jongmans et al. ,1997)。生长在前苏 联切尔洛贝尔核电站的高核辐射中心室墙上的 37 种半知菌菌物 (Zhdanova et al. ,2000) , 提示了菌物 分解作用在废墟重建和环境保护中的又一个生态作 用, 这说明菌物的分解作用如同菌物的共生作用一 样，在生态恢复、退耕还林 (草)、废墟造林等方面都 有其应用的前景 ( Ba \& Guissou, 1996;Cruz et al. , 2000 ;Ferris et al. 2000)。另外, 由于菌物在森林生 态中碳、氮循环中的作用, 研究菌物对我国环境生态 的碳、氮汇研究是必须的。

\section{参 考 文 献}

Alexopoulos, C. J., C. W. Mims \& M. Blackwell (translated by Yao, Y. J. (姚一建), Y. Li (李玉), K. Q. Zhang (张克 勤), M. L. Wu (吴美丽), Y. Q. Huang (黄永青), Y. Z. Wang (王也珍)，W. Q. Chen (陈伟群)，F. Y. Bai (白逢 彦)，Z. Y. Liu (刘作易)，Z. Q. Yuan (袁自清)，Z. Wang (王征), G. Z. Lu (吕国忠), Q. X. Wu (吴秋欣), T. H. Li (李泰辉)，Y.C. Dai (戴玉成)，Y.C. Niu (牛永春), L. Guo (郭林), Z. D. Jiang (姜子德), Z. H. Wang (王宗 华), S. L. Chen (陈双林), Q. Wang (王琦) \& S. Y. Guo (郭守玉)). 2002. Introductory mycology. 4th ed. Beijing: China Agricultural Press. 1 771. (in Chinese)

Arocena, J. M. \& K. R. Glowa. 2000. Mineral weathering in ectomycorrhizosphere of subalpine fir (Abies lasiocarpa (Hook.) Nutt. ) as revealed by soil solution composition. Forest Ecology and Management, 133: $61 \sim 70$.

Ba, A. M. \& T. Guissou. 1996. Rock phosphate and vesicular-arbuscular mycorrhiza effects on growth and nutrient uptake of Faidherbia albida (Del.) seedlings in an alkaline sandy soil. Agroforestry Systems, 34: 129 137 .

Berbee, M. L. \& J. W. Taylor. 2000. Systematics and evolution. In: McLaughlin, D. J. \& E. McLaughlin eds. The mycota. Vol. VII B. New York: Springer-Verlag. 229 246.

Bergelson, J. M. \& M. J. Crawley. 1988. Mycorrhizal infection and plant species diversity. Nature, 334: 202.

Bidartondo, M. I., H. Ek., H. Wallander \& B. Söderström. 2000. Do nutrient additions alter carbon sink strength of ectomycorrhizal fungi? New Phytologist, 151: 543 550.

Blackwell, M., R. Vilgalys \& J. W. Taylor. 2001. Fungi. http://tolweb. org/tree/eukaryotes/fungi/fungi. html.

Blackwell, M. 2000. Terrestrial life-fungal from the start? Science, 289: $1884 \sim 1885$

Brown, J. H., T. G. Whitham, S. K. M. Ernest \& G. Catherine. 2001. Complex species interactions and the dynamics of ecological systems: long-term experiments. Science, 293: $643 \sim$ 664.

Burke, R. M. \& J. W. G. Cairney. 1997. Carbohydrolase pro- 
duction by the ericoid mycorrhizal fungus Hymenoscyphus ericae under solid-state fermentation conditions. Mycological Research, 101: $1135 \sim 1139$.

Burke, R. M. \& J. W. G. Cairney. 1998. Carbohydrate oxidases in ericoid and ectomycorrhizal fungi: a possible source of Fenton radicals during the degradation of lingnocellulose. New Phytologist, 139: $637 \sim 645$.

Cairney, J. W. G. 1999. Intraspecific physiological variation: implications for understanding functional diversity in ectomycorrhizal fungi. Mycorrhiza, 9: $125 \sim 135$.

Chapin III, F. S., B. H. Walker, R. J. Hobbs, D. U. Hooper, J. H. Lawton, O. E. Sala \& D. Tilman. 1997. Biotic control over the functioning of ecosystems. Science, 277: $500 \sim 513$.

Clark, R. B., R. W. Zobel \& S. K. Zeto. 1999. Effects of mycorrhizal fungus isolates on mineral acquisition by Panicum virgatum in acidic soil. Mycorrhiza, 9: $167 \sim 176$.

Clay, K. \& J. Holah. 1999. Fungal endophyte symbiosis and plant diversity in successional fields. Science, 285: $1742 \sim 1744$.

Cruz, A. F., T. Ishii \& K. Kadoya, 2000. Effects of arbuscular mycorrhizal fungi on tree growth, leaf water potential, and levels of 1-aminocyclopropane-1-carboxylic acid and ethylene in the roots of papaya under water-stress conditions. Mycorrhiza, 10: $121 \sim 123$.

Cullings, K. W., T. M. Szaro \& T. D. Bruns. 1996. Evolution of extreme specialization within a lineage of ectomycorrhizal epiparasites. Nature, 379: $63 \sim 66$.

Dahlberg, A. 2001. Community ecology of ectomycorrhizal fungi: an advancing interdisciplinary field. New Phytologist, 150: 555 $\sim 562$.

Feng, Z. W. (冯宗炜) , X. K. Wang (王效科) \& G. Wu (吴 刚). 1999. The forest ecosystem's biomass and production in China. Beiiing: Science Press. 5 6. (in Chinese)

Ferris, R., A. J. Peace \& A. C. Newton. 2000. Macrofungal communities of lowland Scots pine (Pinus sylvestris L.) and Norway spruce (Picea abies (L.) Karsten.) plantations in England: relationships with site factors and stand structure. Forest Ecology and Management, 131: $255 \sim 267$.

Finlay, R. D. \& B. Söderström. 1998. Mycorrhiza and carbon flow to the soil. In: Allen, M. ed. Mycorrhiza functioning. London: Chapman \& Hall. $134 \sim 160$.

Finlay, R. D., H. Ek, G. Odham \& B. Söderström. 1998. Mycelial uptake translocation and assimilation of nitrogen from ${ }^{15} \mathrm{~N}$-labeled ammonium by Pinus sylvestris plants infected with four different ectomycorrhizal fungi. New Phytologist, 110: 59 66.

Gargas, A., P. T. DePrist \& M. Grube. 1995. Multiple origins of lichen symbioses in fungi suggested by SSU rDNA phylogeny. Science, 268: $1492 \sim 1495$.

Gerlitz, T. G. B. \& W. B. Werk. 1994. Investigations on phosphate uptake and polyphosphate metabolism by mycorrhized and non-mycorrhized roots of beech and pine as investigated by in vivo ${ }^{31} \mathrm{P}-\mathrm{NMR}$. Mycorrihza, 4: $207 \sim 214$.

Gerliz, T. G. M. 1996. Effects of alumium on polyphosphate mobilization of the ectomycorrhizal fungus Suillus bovinus. Plant and Soil, 178: $133 \sim 140$.

Gleich, M. (translated by Ma, J. (马剑), N. He (何宁), L. Y. Zhong (钟礼译), Y. Zhang (张渝) \& H. Y. Xu (许红 燕)). 2001. Life count. Guiyang: Guizhou Science and Technology Publishing House. 265. (in Chinese)

Gong, M. Q. (弓明钦) , Y. L. Chen (陈应龙) \& Z. L. Zhong (仲崇禄). 1997. Mycorrhizal research and application. Beijing: China Forestry Publishing House. 1 196. (in Chinese)

Hao, S. G. (郝守刚), X. P. Ma (马学平) \& X. P. Dong (董 熙平). 2000. The origin and evolution of life - life in earth history. Beijing: China Higher Education Press. 87 104. (in Chi- nese)

Harley, J. L. \& S. E. Smith. 1983. Mycorrhizal symbiosis. New York: Academic Press. $23 \sim 29$.

Hawksworth, L. D. 1991. The fungal dimension of biodiversity: magnitude, significance, and conservation. Mycological Research, 95: $641 \sim 655$.

Hecht, J. 1993. Animals and fungi closer than anyone expected. New Scientist, 138: 16.

Heckman, D. S. , D. M. Geiser, B. R. Eidell, R. L. Stauffer, N. L. Kardos \& S. B. Hedges. 2001. Molecular evidence for the early colonization of land by fungi and plants. Science, 293: $1129 \sim 1133$.

Helgason, T., T. J. Daniell, R. Husband, A. H. Fitter \& J. P. W. Young. 1998. Ploughing up the wood-wide web? Nature, 394: 431 .

Hobbie, E. A., N. S. Weber \& J. M. Trappe. 2001. Mycorrhizal vs saprotrophic status of fungi: the isotopic evidence. New Phytologist, 150: $601 \sim 610$.

Hogberg, P., A. Nordgren, N. Buchmann, A. F. S. Taylor, A. Ekblad, M. N. Hogberg, G. Nyberg, M. Ottosson-Lofveniun \& D. J. Read. 2001. Large-scale forest girdling shows that current photosynthesis drives soil respiration. Nature, 411: $789 \sim 792$.

Hogberg, P., A. H. Plamboeck, A. F. S. Taylor \& P. M. A. Fransson. 1999. Natural ${ }^{13} \mathrm{C}$ abundance reveals trophic status of fungi and host-origin of carbon in mycorrhizal fungi in mixed forests. Proceedings of National Academy of Science of the United States of America, 96: $8534 \sim 8539$.

Janzen, D. H. 1970. Herbivores and the number of tree species in tropical forests. The American Naturalist, 104: $501 \sim 528$.

Jiang, Y. X. (蒋有绪), Q. S. Guo (郭泉水) \& J. Ma (马 鹃) . 1998a. The forest community's classification and characteristics in China. Beijing: Science Press. $21 \sim 178$. (in Chinese)

Jiang, Y. X. (蒋有绪) \& J. P. Lu (卢俊培). 1998b. The study on tropical forest ecosystem in Hainan Island, China. Beijing: China Forestry Publishing House. 156. (in Chinese)

Jongmans, A. G., N. van Breemen, U. Lundstrom, P. A. W. van Hees, R. D. Finlay, M. Srinivasan, T. Unestam, R. Giesler, P. A. Melkerud \& M. Olsson. 1997. Rock-eating fungi. Nature, 389: $682 \sim 683$.

Kohn, L. M. 1999. The fascination of fungi. Science, 83: $644 \sim$ 647 .

Komonen, A., R. Penttila, M. Lindgren \& I. Hanski. 2000. Forest fragmentation truncates a food chain based on an oldgrowth forest bracket fungus. Oikos, 90: 119 126 .

Lamhamedi, M. S., C. Godbout \& J. A. Fortin. 1994. Dependence of Laccaria bicolor basidiome demelopment on current photosynthesis of Pinus strobes seedlings. Candian Journal of Forest Research, 24: $1797 \sim 1804$.

Lowe, A., W. Einig, L. Shi, P. Dizengremel \& R. Hamp. 2000. Mycorrhization and elevated $\mathrm{CO}_{2}$ both increase the capacity for sucrose synthesis in source leaves of spruce and aspen. New Phytologist, 145: $565 \sim 574$.

Lutzoni, F., M. Pagel \& V. Reeb. 2001. Major fungal lineages are derived from lichen symbiotic ancestors. Nature, 411: 937 940 .

MacFall, J. S., S. A. Slack \& S. Wehrli. 1992. Phosphorus distribution in red pine roots and the ectomycorrhizal fungus Heleloma arenosa. Plant Physiology, 100: $713 \sim 717$.

Marschner, H. \& B. Dell. 1994. Nutrient uptake in mycorrhizal symbiosis. Plant and Soil, 159: 89 102 .

Martin, F. 1985. ${ }^{15} \mathrm{~N}-\mathrm{NMR}$ studies of nitrogen assimilation and amino acid biosynthesis in ectomycorrhizal fungus Cenococcum graniforme. FEBS Letters, 182: $350 \sim 354$.

Martin, F., P. Rubini, R. Cote \& I. Kottke. 1994. Aluminum 
polyphosphate complexes in mycorrhizal basidiomycete Laccaria bicolor: a ${ }^{27}$ AI-nuclear magnetic resonance study. Planta, 194 : $241 \sim 246$

Naeshom, T., A. Ekblad, A. Nordin, R. Giesler, M. Hogberg \& P. Hogberg. 1998. Boreal forest plants take up organic nitrogen. Nature, 392: $914 \sim 916$.

Nehls, U., S. Mikolajewski, E. Magel \& R. Hampp. 2001. Carbohydrate metabolism in ectomycorrhizas: gene expression, monosaccharide transport and metabolic control. New Phytologist, 150: $533 \sim 541$.

News Centre, the Chinese Academy of Forest (中国林业科学研 究院新闻中心). 2001. ASEM symposium on forest conservation and sustainable development, Guiyang city, Guizhou province, 16-20 July 2001. http://nic6. forestry. ac.cn/newcaf/ detaill.$c f m$ ? file $=323$. (in Chinese)

Pacific Northeast Research Statin/USDA Forest Sevice. 2000. News and information. http//www.fs. fed.us/pnw/news/fungus. htm.

Packer, A. \& K. Clay. 2000. Soil pathogens and spatial patterns of seedling mortality in a temperate tree. Nature, 404: 278 281 .

Pain, S. 1999. Fiendish fungus: a hybrid blight is running amok in Europe's woodlands. New Science, 15: 7.

Paine, T. D., K. F. Raffa \& T. C. Harrington. 1997. Interactions among scolytid bark beetles, their associated fungi, and live host conifer. Annual Review of Entomology, 42: 179 206.

Pennisi, E. A. 2001. Molecular approach to mushroom hunting. Science, 293: $1027 \sim 1028$.

Pfeffer, P. E., B. Bago \& Y. Shachar-Hill. 2001. Exploring mycorrhizal function with NMR spectroscopy. New Phytologist, 150: $543 \sim 553$

Pirozynski, K. A. \& D. W. Malloch. 1975. The origin of land plants: a matter of mycotrophism. Biosystems, 6: 153 164 .

Pugnaire, F. I. 2001. Variability of inorganic nutrient concentrations in leaves. New Phytologist, 150: $506 \sim 507$.

Read, D. 1997. The ties that bind. Nature, 388: $517 \sim 518$.

Reddel, T., Y. Yang \& W. A. Shipton. 1997. Cluster roots and mycorrhizae in Casurina cunninghamiana: their occurrence and formation in relation to phosphorous supply. Australian Journal of Botany, 45: $41 \sim 51$.

Redecker, D., R. Kodner \& L. E. Graham. 2000. Glomalean fungi from the Ordovician. Science, 289: $1920 \sim 1921$.

Rogers, J. B., A. S. Laidlaw \& P. Christie. 2001. The role of arbuscular mycorrhizal fungi in the transfer of nutrients between white clover and perennial ryegrass. Chemosphere, 242: 153 159 .

Saikkonen, K., S. H. Faeth, M. Helander \& T. J. Sullivan. 1998. Fungal endophytes: a continuum of interactions with host plants. Annual of Review Ecology System, 29: $319 \sim 343$.

Setala, H., P. Kulmala, J. Mikola \& A. M. Markkola. 1999. Influence of ectomycorrhiza on the structure of detrital food webs in pine rhizosphere. Oikos, 87: $113 \sim 122$.

Simard, S. W., D. A. Perry, M. D. Jones, D. D. Myrold, D. M. Durall \& R. Molina. 1997. Net transfer of carbon between ectomycorrhizal tree species in the field. Nature, 388: 579 582 .

Smith, S. E. \& D. J. Read. 1997. Mycorrhizal symbiosis, 2th ed. San Diego: Academic Press. 11 56 .

Söderström, B. 1992. The ecological potential of the ectomycorrhizal mycelium. In: Read, D. J., D. H. Lewis, A. H. Fitter \& I. J. Alexander eds. Mycorrhizas in ecosystems. Walingford: CAB International. $77 \sim 83$.

Stitt, M. 1991. Rising $\mathrm{CO}_{2}$ levels and their potential significance for carbon flow in photosynthetic cells. Plant, Cell and Environment, 14: $741 \sim 762$.

Taylor, A. F. S., L. Hogbom, M. Hogberg, A. J. E. Lyon, T. Nasholm \& P. Hogberg. 1997. Natural ${ }^{15} \mathrm{~N}$ abundance in fruit bodies of ectomycorrhizal fungi from boreal forests. New Phytologist, 136: $713 \sim 720$.

Turnbull, E. 1995. Not only nuts in May. Mycologist, 9: $82 \sim 83$. van der Heijden, M. G. A., J. N. Klironomos, M. Ursic, P. Moutoglis, R. Streitwolf-Engel, T. Boller, A. Wiemken \& L. R. Sanders. 1998. Mycorrhizal fungal diversity determines plant biodiversity, ecosystem variability and productivity. Nature, 396: $69 \sim 72$.

van der Putten, W. H. 2000. Pathogen-driven forest diversity. Nature, 404: $232 \sim 233$.

Wei, J. C. (魏江春). 1993. Biological diversity and systemaricalness of panomycetes, and their significance to the development of human beings. Chinese Biodiversity (生物多样性), 1:23 25. (in Chinese with English abstract)

Wu, B., K. Nara \& T. Hogetsu. 2001. Can ${ }^{14}$ C-labeled photosynthetic products move between Pinus densiflora seedlings linked by ectomycorrhiza mycelia? New Phytologist, 149: $137 \sim 146$.

Zhdanova, N. N., V. A. Zakharchenko, V. V. Vember \& B. T. Nakonechnaya. 2000. Fungi from Chernobyl. Mycological Research, 104: $1421 \sim 1426$. 